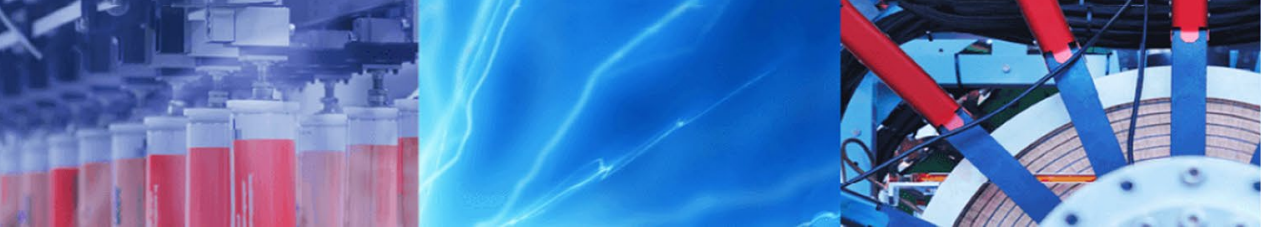

Research Article

\title{
Effects of radiation and magnetohydrodynamics on heat transfer of nanofluid flow over a plate
}

\author{
Amireh Nourbakhsh ${ }^{1} \cdot$ Hamdolah Mombeni ${ }^{1} \cdot$ Morteza Bayareh $^{2}$
}

Received: 27 August 2019 / Accepted: 4 November 2019 / Published online: 9 November 2019

(c) Springer Nature Switzerland AG 2019

\begin{abstract}
The purpose of this study is to investigate the effects of radiation and magnetohydrodynamics on heat transfer of a nanofluid flow over a plate under constant heat flux or constant temperature. The effects of magnetohydrodynamics and radiation on the wall are assumed to be uniform and the plate is static. Momentum and energy equations are solved using the Crank-Nicholson finite difference method. In this paper, the effect of parameters such as Prandtl number, radiation parameter, magnetohydrodynamic parameter on velocity, temperature, Nusselt number and friction coefficients is investigated. The results demonstrated that an increase in magnetohydrodynamic parameter results in an increase in fluid surface penetration in the vicinity of the wall. The temperature gradient increases with the radiation parameter, leading to an increase in the fluid motion.
\end{abstract}

Keywords Static flow · Nanofluid · Magnetohydrodynamics · Radiation effects · Vertical plate

\section{Introduction}

One of the topics of interest in new technologies is heat transfer in the Magnetohydrodynamics (MHD) systems. MHD describes the interaction between fluid and magnetic field. The problems related to nanofluids on the plate and heat transfer in the presence of the magnetic field have many applications in engineering sciences, including in the aerospace industry, turbomachines, MHD pumps, chemical and petrochemical engineering, MHD power generators, heat exchangers, flow meters, electronics, chemistry, nuclear reactors, and combustion systems. Numerous investigations have been done on the laminar flow over a vertical plate. These studies can be divided into natural convection, forced convection, and mixed convection with symmetric or asymmetric heating when the plates have constant temperature or heat flux. In general, fully developed flows have been studied numerically. Thermo-MHD flow over different geometries has many practical applications due to that the magnetic field improves the control of heat transfer rate. Thus, thermos-MHD is commonly employed in technological process such as metallurgical processes, transpiration cooling of hypersonic airplanes, production of semiconductors, etc. Heat transfer can be controlled by thermal MHD flows: these flows can increase or suppress the heat transfer.

Sattar et al. [1] studied free convection heat and mass transfer on a vertical porous plate in a porous medium with variable suction rate. Soundalgekar et al. [2] investigated free convective heat transfer of an unstable flow passing through a vertical plate with constant suction and mass transfer. They used a finite difference numerical method to discrete the dimensionless equations.

Raptis and Kafousia [3] studied heat transfer in porous media with a uniform MHD flow on a vertical plate. Ghokhale [4] investigated free convection heat transfer with a uniform MHD flow on a vertical porous plate under constant heat flux conditions. Ahmad [5] studied the free

$\triangle$ Amireh Nourbakhsh, nourbakhsh@basu.ac.ir | 'Department of Mechanical Engineering, Bu-Ali Sina University, Hamedan, Iran. ${ }^{2}$ Department of Mechanical Engineering, Shahrekord University, Shahrekord, Iran. 
convection heat transfer coupled with the uniform MHD effect of a non-Newtonian power law fluid on a cold plate using constant temperature boundary condition. He did not consider the porosity effects and solved the governing equations numerically.

Naby et al. [6] studied the effects of radiation on free convection heat transfer with uniform MHD on a vertical porous plate. Kim [7] studied free convection heat transfer with the effect of MHD for the power law fluid flow on a vertical porous plate in a porous medium. Sherman and Singh [8] investigated free convection heat transfer coupled with mass transfer on a vertical porous plate with variable suction and heat source. After obtaining the similarity parameters, they were placed in momentum, energy, and mass transfer equations. They obtained a series of dimensionless equations and solved them using the Rang Kuta numerical method and Shooting method. Takhar et al. [9] studied free convection heat transfer on a vertical plate with unlimited length and variable surface temperature. Suundalgekar et al. [10] investigated the effects of free convection heat transfer and mass transfer along a vertical plate on the uniform magnetohydrodynamic flow applied to the plate. Sacheti et al. [11] studied an exact solution for the free convection flow coupled with the magnetohydrodynamic effect
Isman et al. [17] for the first time used the concept of nanotechnology as an obstacle in earlier centuries using the unique properties of nanoparticles. Solid nanoparticles are also ultrafine $(100 \mathrm{~nm})$ and can be considered as fluid.

In this paper, two-dimensional free convection heat transfer of nanofluids is considered. In order to increase the heat transfer, a uniform external magnetic field is applied perpendicular to the flow. In this problem, the governing equations are non-dimensionalized and solved numerically. The main goal of the present paper is to find the influence of MHD and radiation on velocity, temperature, Nusselt number, and friction coefficient

\section{Governing equations}

The governing equations for incompressible fluid are continuity, momentum and energy equations $[18,19]$ :

Continuity equation:

$$
\frac{\partial u}{\partial x}+\frac{\partial v}{\partial y}+\frac{\partial w}{\partial z}=0
$$

Momentum equation:

$\rho_{n f}\left(\frac{\partial u}{\partial t}+u \frac{\partial u}{\partial x}+v \frac{\partial u}{\partial y}+w \frac{\partial u}{\partial z}\right)=-\frac{1}{\rho} \frac{\partial p}{\partial x}+\mu_{n f}\left(\frac{\partial^{2} u}{\partial x^{2}}+\frac{\partial^{2} u}{\partial y^{2}}+\frac{\partial^{2} u}{\partial z^{2}}\right)$
$\rho_{n f}\left(\frac{\partial v}{\partial t}+u \frac{\partial v}{\partial x}+v \frac{\partial v}{\partial y}+w \frac{\partial v}{\partial z}\right)=-\frac{1}{\rho} \frac{\partial p}{\partial y}+\mu_{n f}\left(\frac{\partial^{2} v}{\partial x^{2}}+\frac{\partial^{2} v}{\partial y^{2}}+\frac{\partial^{2} v}{\partial z^{2}}\right)$
$\rho_{n f}\left(\frac{\partial w}{\partial t}+u \frac{\partial w}{\partial x}+v \frac{\partial w}{\partial y}+w \frac{\partial w}{\partial z}\right)=-\frac{1}{\rho} \frac{\partial p}{\partial z}+\mu_{n f}\left(\frac{\partial^{2} w}{\partial x^{2}}+\frac{\partial^{2} w}{\partial y^{2}}+\frac{\partial^{2} w}{\partial z^{2}}\right)$

under constant wall heat flux conditions. Shanker and Kishan [12] investigated the effects of mass transfer on magnetohydrodynamic flow on a vertical plate with variable wall temperature or variable wall heat flux as the plate moves suddenly. Elbashbeshy [13] studied heat and mass transfer on a vertical plate with variable surface temperature under magnetohydrodynamic effect. Helmy [14] investigated the unsteady free convection heat transfer on a vertical porous plate. Takhar et al. [15] studied unsteady mixed convection heat transfer in a rotating vertical cone with magnetohydrodynamic flow. Ganesan and Palani [16] studied numerically the unsteady free convection flow on a vertical surface with a variable surface temperature under a magnetohydrodynamic effect. Although Maxwell's theory is outdated, it can be upgraded using the idea of nanoparticles to provide more basic fluid stability. This is a great opportunity to apply nanotechnology to thermal engineering.
Energy equation:

$\left(\rho C_{p}\right)_{n f}\left(\frac{\partial T}{\partial t}+u \frac{\partial T}{\partial x}+v \frac{\partial T}{\partial y}+w \frac{\partial T}{\partial z}\right)=k_{n f}\left(\frac{\partial^{2} T}{\partial x^{2}}+\frac{\partial^{2} T}{\partial y^{2}}+\frac{\partial^{2} T}{\partial z^{2}}\right)$

Table 1 shows the therophysical properties of water and nanofluids. There are several papers about the prediction of thermophysical properties of nanofluids [20-22].

Density, dynamic viscosity, and thermal conductivity for nanofluids based on the properties of base fluid and nanoparticles are expressed as follows [23]:

$$
\begin{aligned}
& \rho_{n f}=(1-\phi) \rho_{f}+\phi \rho_{s}, \quad\left(\rho C_{P}\right)_{n f}=(1-\phi)\left(\rho C_{P}\right)_{f}+\phi\left(\rho C_{P}\right)_{s} \\
& \mu_{n f}=\frac{\mu_{f}}{(1-\phi)^{2.5}}, \quad \frac{k_{n f}}{k_{f}}=\frac{k_{s}+2 k_{f}-2 \phi\left(k_{f}-k_{s}\right)}{k_{s}+2 k_{f}+2 \phi\left(k_{f}-k_{s}\right)}
\end{aligned}
$$


Table 1 Therophysical properties of water and nanofluids

\begin{tabular}{|c|c|c|c|c|c|c|}
\hline Property & Test instruments & Water & $\mathrm{Cu}$ & $\mathrm{Ag}$ & $\mathrm{Al}_{2} \mathrm{O}_{3}$ & $\mathrm{TiO}_{2}$ \\
\hline$C_{P}(\mathrm{~J} / \mathrm{Kg} \mathrm{K})$ & $\begin{array}{l}\text { Hot disk—Dynalene-differential scanning calorimeter (DSC)—Laser Flash Technique } \\
\text { (LFT) }\end{array}$ & 4179 & 385 & 235 & 765 & 686.2 \\
\hline$\rho\left(\mathrm{Kg} / \mathrm{m}^{3}\right)$ & Hydrometer (for water)—Eq. 4 is used to calculate the nanofluid density & 997.1 & 8933 & 10,500 & 3970 & 4250 \\
\hline$k(\mathrm{~W} / \mathrm{m} \mathrm{K})$ & $\begin{array}{l}\text { Thermal conductivity meters: TLS-100, THW-L2, TPS-EFF, GFHM-02 and TPS-M1, Light- } \\
\text { Flash analyzer }\end{array}$ & 0.613 & 401 & 429 & 40 & 9.9538 \\
\hline$\beta \times 10^{-5}\left(\mathrm{~K}^{-1}\right)$ & Time domain thermoreflectance_-laser flash/xenon flash—transient hot bridge & 21 & 1.67 & 1.89 & 0.85 & 0.9 \\
\hline
\end{tabular}

where $\Phi$ is the volume fraction of nanoparticles. The subtypes $f$ and $s$ refer to the solid and fluid, respectively.

\section{Governing equations under magnetohydrodynamic and radiation effects}

The governing equations under magnetohydrodynamic and radiation effects are presented in this section. It should be noted that the viscosity dissipation is considered in the energy equation. Further simplifications are: (A) first- and second-order derivatives of $u, v$, and $T$ are zero and.

(B) the derivative of pressure relative to $x, y$, and $z$ are assumed to be zero.

Using the above simplifications, the equations of continuity, momentum, and energy are as follows, respectively: field strength, $\sigma$ is electrical conductivity coefficient, $C_{p}$ is heat transfer coefficient at constant pressure, $q_{r}$ is thermal radiation, $\sigma^{*}$ is Stephen Boltzmann constant, $k^{*}$ is Rutland absorption coefficient, $v_{n f}$ is nanofluid kinematic viscosity, $\beta$ is heat expansion coefficient of Nanofluids, $\rho$ is fluid density, $T_{\infty}$ is free-flow temperature, and $g$ is gravity acceleration.

Also, using the nanofluid model, thermal conductivity of nanofluid can be written as follows:

$k_{n f}=k_{f} \frac{k_{s}+2 k_{f}-2 \phi\left(k_{f}-k_{s}\right)}{k_{s}+2 k_{f}+2 \phi\left(k_{f}-k_{s}\right)}$

And the thermo-physical properties of nanofluid can be described as:

$\left(\rho C_{P}\right)_{n f}=(1-\phi)\left(\rho C_{P}\right)_{f}+\phi\left(\rho C_{P}\right)_{s}$

Thus, the energy equation is:

$$
\begin{aligned}
& \frac{\partial T}{\partial t}+u \frac{\partial T}{\partial x}+v \frac{\partial T}{\partial y}+w \frac{\partial T}{\partial z}= \\
& \quad \frac{k_{s}+2 k_{f}-2 \phi\left(k_{f}-k_{s}\right)}{k_{s}+2 k_{f}+2 \phi\left(k_{f}-k_{s}\right)} \frac{k_{f}}{\left[(1-\phi)\left(\rho C_{P}\right)_{f}+\phi\left(\rho C_{P}\right)_{s}\right]}\left[\frac{\partial^{2} T}{\partial z^{2}}-\frac{16 \sigma^{*} T_{\infty}^{3}}{3 k^{*}}\left(\frac{\partial^{2} T}{\partial z^{2}}\right)\right]
\end{aligned}
$$

$\frac{\partial u}{\partial x}+\frac{\partial v}{\partial y}+\frac{\partial w}{\partial z}=0$

$\frac{\partial u}{\partial t}+u \frac{\partial u}{\partial x}+v \frac{\partial u}{\partial y}+w \frac{\partial u}{\partial z}=v_{n f}\left(\frac{\partial^{2} u}{\partial z^{2}}\right)-\frac{\sigma B_{0}^{2}}{\rho_{n f}} u$

$\frac{\partial w}{\partial t}+u \frac{\partial w}{\partial x}+v \frac{\partial w}{\partial y}+w \frac{\partial w}{\partial z}=v_{n f}\left(\frac{\partial^{2} w}{\partial z^{2}}\right)$

$\frac{\partial T}{\partial t}+u \frac{\partial T}{\partial x}+v \frac{\partial T}{\partial y}+w \frac{\partial T}{\partial z}=\bar{\alpha}_{n f} \frac{\partial^{2} T}{\partial z^{2}}-\frac{16 \sigma^{*} T_{\infty}^{3}}{3\left(\rho C_{p}\right)_{n f} k^{*}}\left(\frac{\partial^{2} T}{\partial z^{2}}\right)$

where $\mathrm{u}$ and $\mathrm{v}$ are velocity components in the direction $\mathrm{x}$ and $\mathrm{y} . \mathrm{T}$ is the fluid temperature, $\mathrm{t}$ is time, $B_{0}$ is magnetic
The governing boundary conditions for velocity are as follows:

$z=0: t \leq 0, u=0, v=0, w=0$

$z=0: w=0 v=0, u=0, t>0$

$x=0: w=0, v=0, u=0$

$z \rightarrow \infty: u \rightarrow a x, v=0, w=-2 a z$

The governing boundary conditions for temperature are as follows:

$z=0: T=T_{\infty}, t \leq 0$

$z=0: T=T_{w}, t>0$

$x=0: T=T_{\infty}$ 


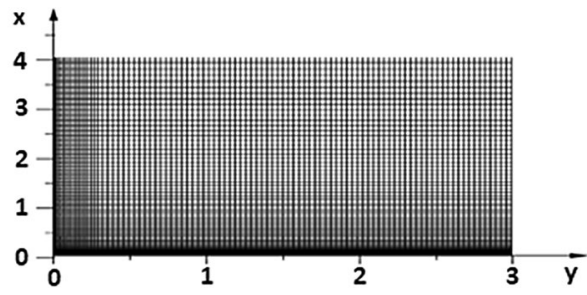

Fig. 1 Computational domain

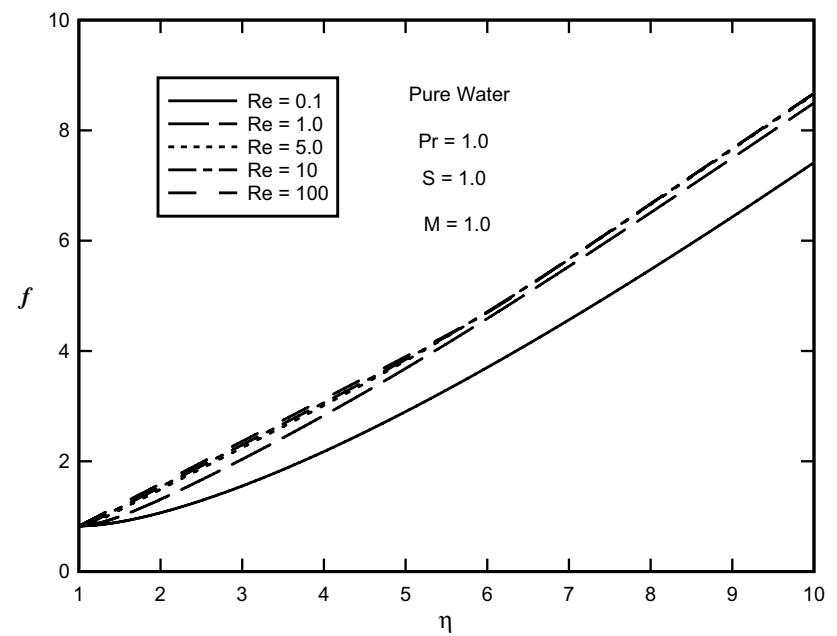

Fig. 2 Velocity profile for different Reynolds numbers

$z \rightarrow \infty: T \rightarrow T_{\infty}$

$z=0: T=T_{\infty}, t \leq 0$

$z=0: \frac{\partial T}{\partial z}=-\frac{q_{w}}{k_{f}}, t>0$

$x=0: T=T_{\infty}$

$z \rightarrow \infty: T \rightarrow T_{\infty}$

Non-dimensional governing parameters are Prandtl number, Reynolds number, and Nusselt number that are defined as follows, respectively:

$\operatorname{Pr}=\frac{v_{f}}{\alpha_{f}}=\frac{(\mu / \rho)_{f}}{\left(k / \rho C_{P}\right)_{f}}=\frac{C_{P} \mu_{f}}{k_{f}}$

$\operatorname{Re}=\frac{a L^{2}}{2 v_{f}}$

$N u=\frac{h \cdot L}{2 k}$

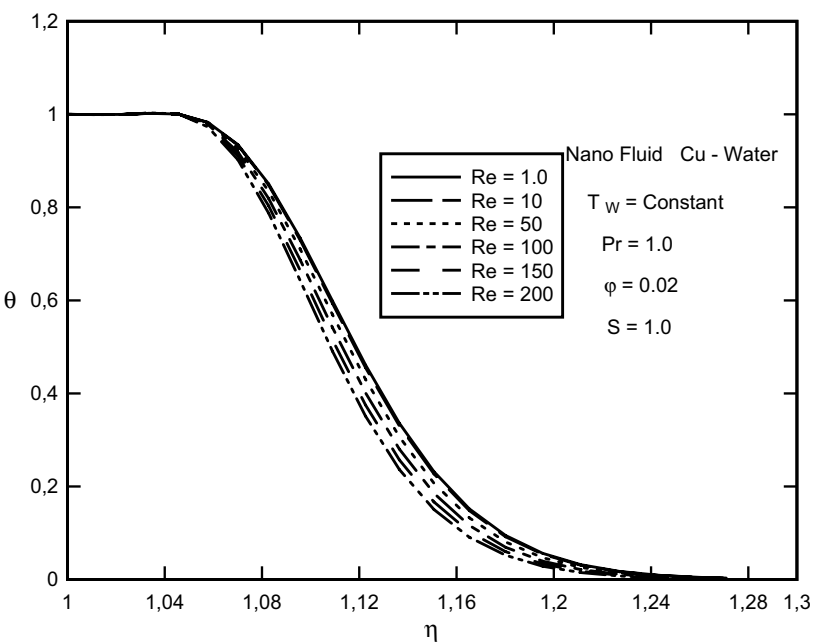

Fig. 3 Dimensionless temperature profile for different Reynolds numbers when $\mathrm{T}_{\mathrm{w}}=$ constant

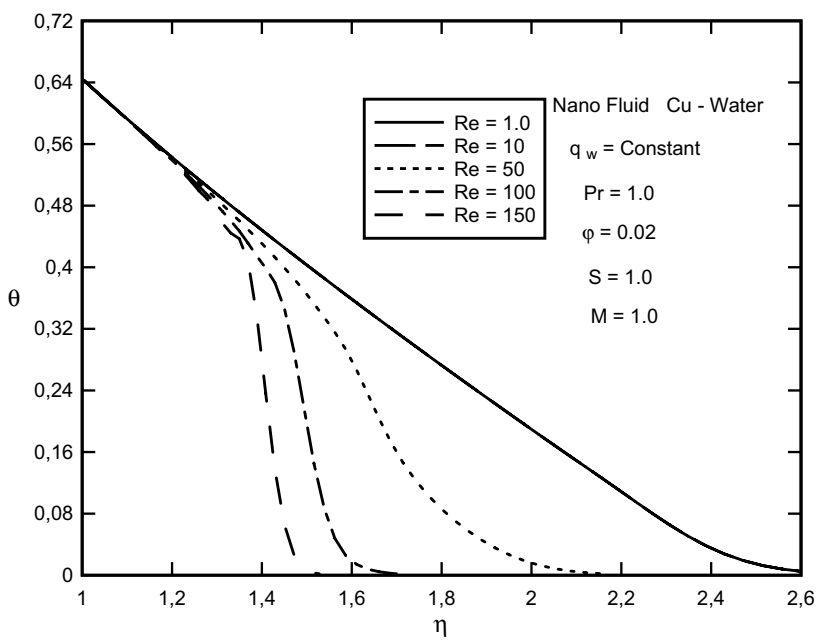

Fig. 4 Dimensionless temperature profile for different Reynolds numbers when $\mathrm{q}_{\mathrm{w}}=$ constant

\section{Results}

The effects of radiation and magnetohydrodynamics on static flow and nanofluid heat transfer on a plate under constant wall flux conditions and constant wall temperature are studied.

The Crank-Nicholson finite difference method is used to solve the governing equations. The effects of parameters such as Prandtl number ( $\mathrm{Pr}$ ), Reynolds number (Re), magnetohydrodynamic parameter $\left(M=\sigma B_{O}^{2} / \rho C_{P}\right)$, radiation parameter $\left(R_{d}=\frac{16 \sigma^{*} T_{\infty}^{3}}{3 k^{*}}\right)$, volume fraction of nanoparticles and fluid surface area (S) on static flow and nanofluid heat transfer are investigated. 


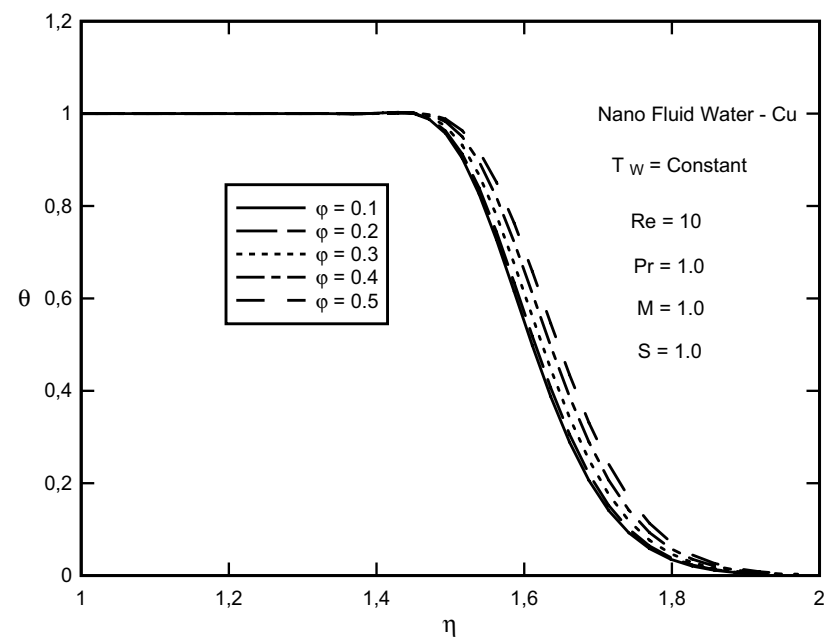

Fig. 5 Dimensionless temperature profile for different volume fractions when $\mathrm{T}_{\mathrm{w}}=$ constant

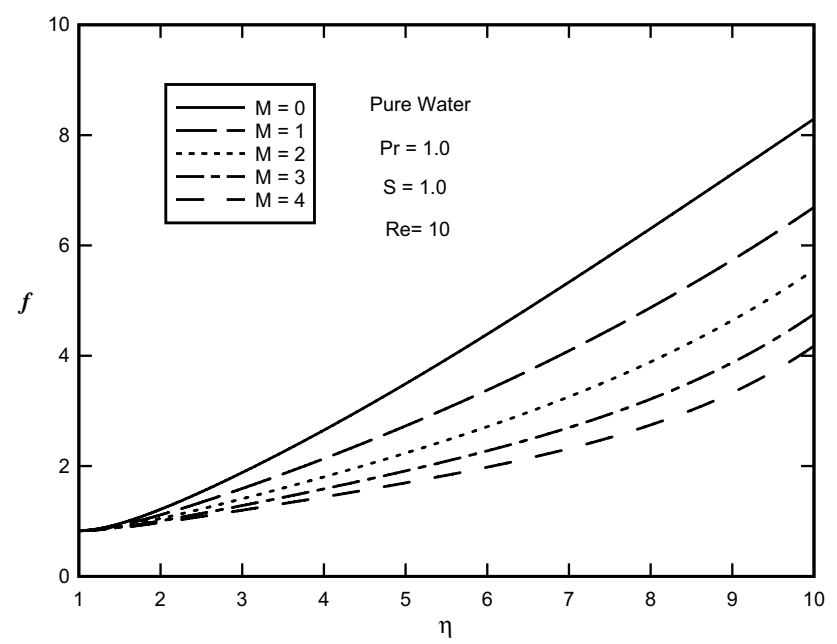

Fig. 6 Velocity profile for different magnetohydrodynamic parameters

Since finite difference method does not need symmetric grid and the temperature and velocity gradients near the wall are high, finer grid is used in left and bottom surfaces (Fig. 1).

Figures 2, 3 and 4 show the temperature and velocity profiles at different Reynolds numbers for water/ copper nanofluid and pure water, where constant surface temperature and constant heat flux conditions are assumed. Other parameters are: $\operatorname{Pr}=1, \mathrm{~S}=1$, and $\phi=0.02$. As the Reynolds number increases, the velocity gradient increases, leading to an increase in the acceleration of fluid flow. Therefore, the heat transfer increases. But, the temperature decreases by increasing the Reynolds number. This is due to that as the Reynolds number increases, the temperature gradient between the surface and the fluid

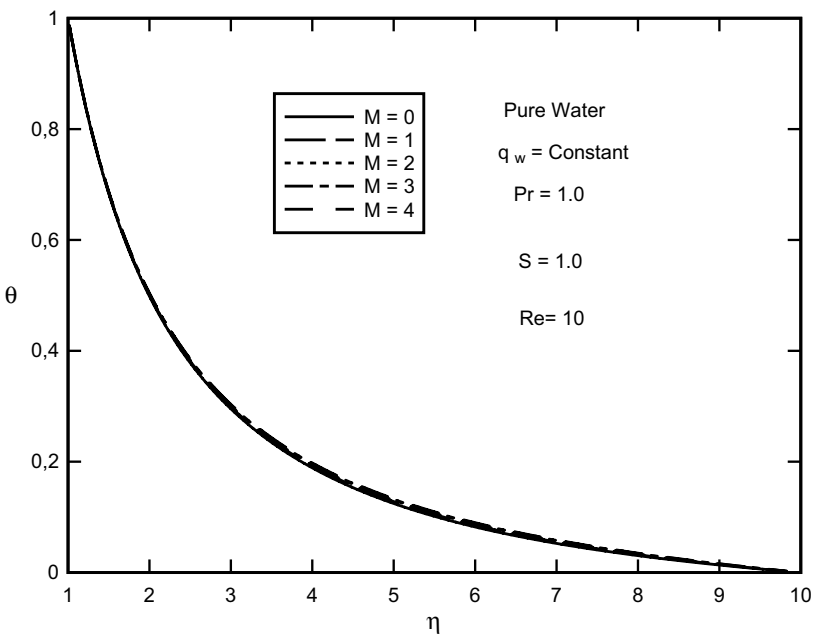

Fig. 7 Dimensionless temperature profile for different magnetohydrodynamic parameters

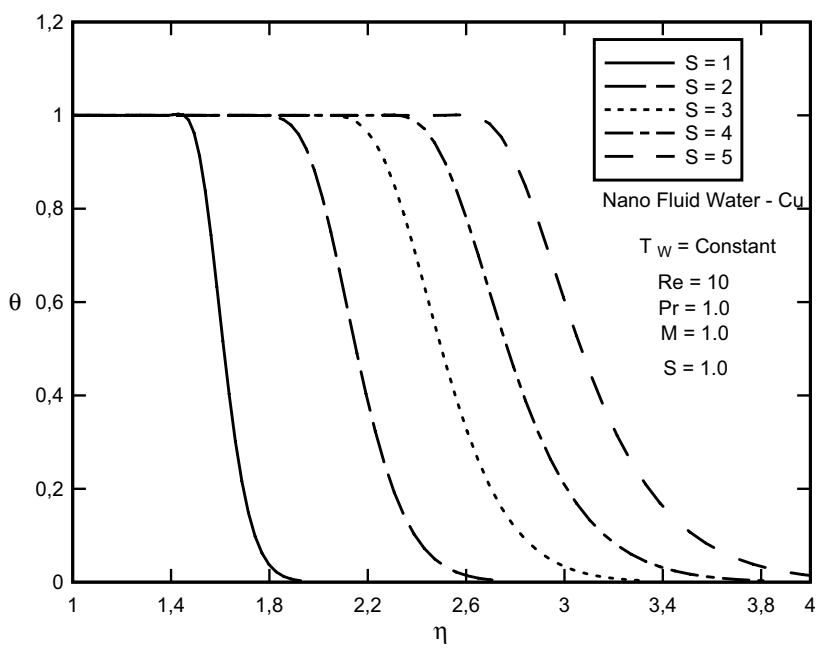

Fig. 8 Velocity profile for different surface wall penetrations

decreases. As a result, the temperature distribution of the fluid decreases.

Figure 5 shows the temperature profile for different volume fractions of nanoparticles for water/copper nanofluids in which constant surface temperature condition is assumed. Other parameters are: $\operatorname{Pr}=1, \mathrm{~S}=1$ and $\operatorname{Re}=10$. As the volume fraction of nanoparticles increases, the fluid velocity decreases. But, increasing the volume fraction of the nanoparticles leads to an increase in the thermal penetration of the fluid near the wall as a result of increasing the temperature value.

Figures 6 and 7 show non-dimensional temperature and velocity profiles for different magnetohydrodynamic parameters. Here, constant surface temperature and constant heat flux are considered for the simulations. Also, 


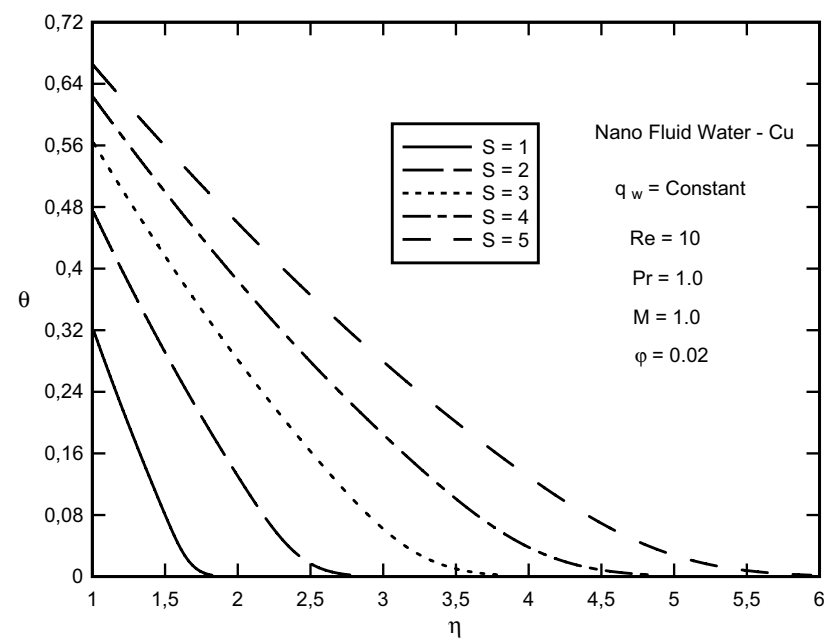

Fig. 9 Dimensionless temperature profile for different surface wall penetrations

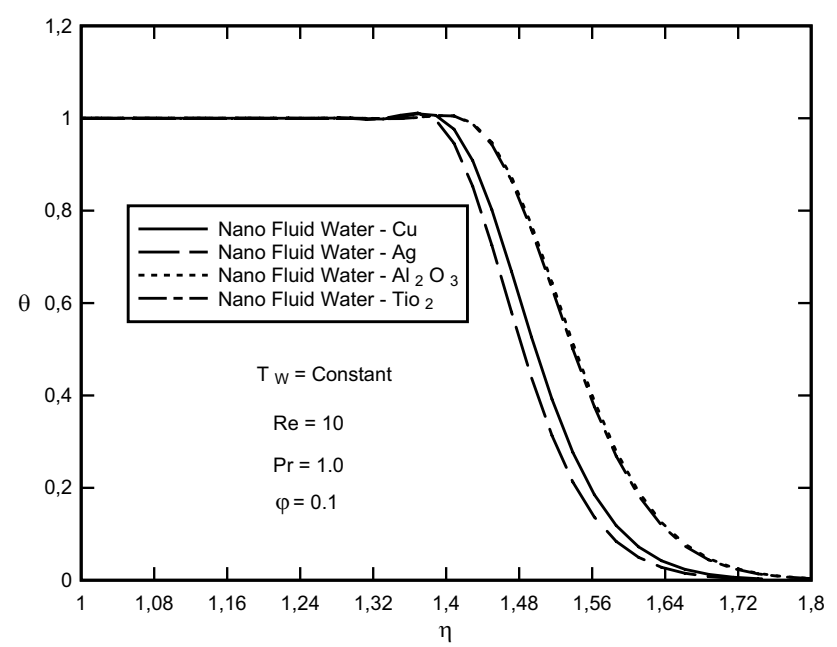

Fig. 10 Dimensionless temperature profile for different nanofluids when $\mathrm{T}_{\mathrm{w}}=$ constant

$\operatorname{Pr}=1, S=1$ and $\operatorname{Re}=10$. Magnetohydrodynamics creates the Lorenz force opposed to the flow direction. Thus, the fluid velocity near the wall decreases, indicating a reduction in the fluid acceleration. As the magnetohydrodynamic parameter increases, the velocity decreases, resulting in a reduction in fluid motion and consequently the velocity distribution decreases. But, an increase in magnetohydrodynamic parameter results in an increase in fluid surface penetration in the vicinity of the wall. Hence, the temperature increases.

Figures 8 and 9 show the temperature profile for different surface wall penetrations of the fluid for the water/ copper nanofluid, in which constant surface temperature

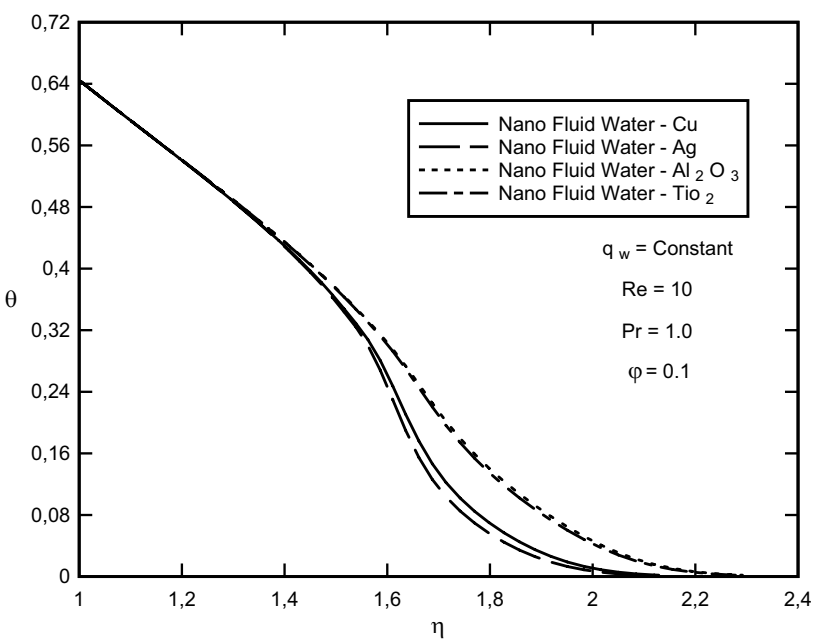

Fig. 11 Dimensionless temperature profile for different nanofluids when $\mathrm{q}_{\mathrm{w}}=$ constant

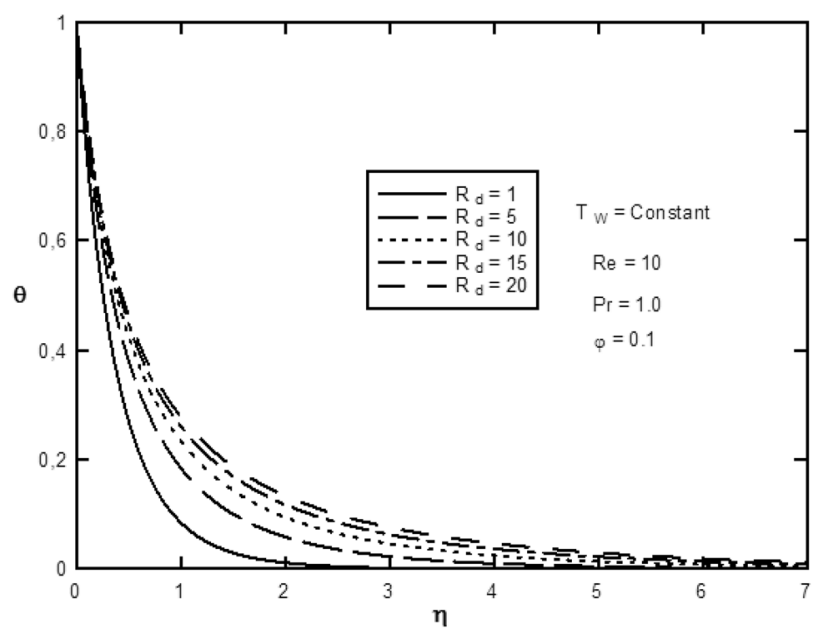

Fig. 12 Dimensionless temperature profile for different radiation parameter when $\mathrm{T}_{\mathrm{w}}=$ constant

and constant heat flux are considered. Also, $\operatorname{Pr}=1, \operatorname{Re}=10$, and $\phi=0.02$. The surface fluid penetration is assumed to be uniform. Thus, as the surface velocity increases, the temperature gradient near the wall increases.

Figures 10 and 11 show the temperature profile for different nanofluids, in which constant surface temperature and constant heat flux are considered. Also, $\operatorname{Pr}=1, \operatorname{Re}=10$, and $\phi=0.02$. As can be seen here, silver nanoparticles have maximum velocity and alumina nanoparticles have minimum velocity. Also, silver nanoparticles have maximum heat transfer and titanium oxide nanoparticles have the minimum one. 


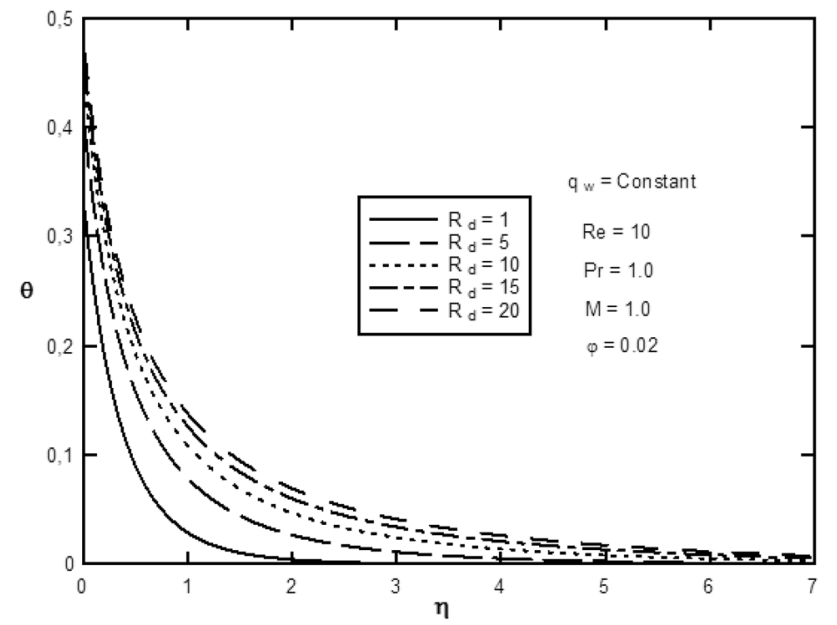

Fig. 13 Dimensionless temperature profile for different radiation parameter when $\mathrm{q}_{\mathrm{w}}=$ constant

Figures 12 and 13 show the temperature profile for different radiation parameters. As the radiation parameter increases, the temperature increases. This is due to that the temperature gradient increases with the radiation parameter, leading to an increase in the fluid motion. Thus, the temperature distribution is increased due to the contribution of the highly fluid heat absorption.

\section{Conclusions}

In this paper, the effects of radiation and magnetohydrodynamics on a static fluid and heat transfer of nanofluid on a plate were investigated. The main results are summarized as follows:

As the Reynolds number increases, the velocity gradient increases and the temperature gradient decreases. It was found that as the volume fraction of nanoparticles increases, the velocity and temperature gradients increase. Increasing the surface penetration of the fluid leads to an increase in the velocity and temperature of the fluid. Silver nanoparticles have the maximum heat transfer and the minimum velocity. The results demonstrated that an increase in magnetohydrodynamic parameter results in an increase in fluid surface penetration in the vicinity of the wall. The temperature gradient increases with the radiation parameter, leading to an increase in the fluid motion.

\section{Compliance with ethical standards}

Conflict of interest The authors declare that they have no conflict of interest.

\section{References}

1. Sattar MA, Rahman MM, Alam MM (2000) Free convection flow and heat transfer through a porous vertical plate immersed in a porous medium with variable suction. J Energy Heat Mass Transf 21:17-21

2. Soundalgekar VM, Wavre PD (1977) Unsteady free convection flow past an infinite vertical plate with constant suction and mass transfer. Int J Heat Mass Transf 20:1363-1373

3. Raptis AA, Kafousias N (1986) Heat transfer in flow through a porous medium bounded by infinite vertical plate under the action of magnetic field. Int J Energy Res 10:97-100

4. Gokhale MY (1991) Magnetohydrodynamic transient-free convection past a semi-infinite vertical plate with constant heat flux. Can J Phys 69:1451-1453

5. Abo-Eldahab EM, Salem AM (2005) MHD free-convection flow of a non-Newtonian power-law fluid at a stretching surface with a uniform free-stream. J Appl Math Comput 169(2):806-818

6. Abd El-Naby MA, Elbarbar EME, AbdElazem NY (2004) Finite difference solution of radiation effects on MHD unsteady freeconvection flow over vertical porous plate. J Appl Math Comput 151:327-346

7. Kim YJ (2001) Unsteady MHD convection flow of polar fluids past a vertical moving porous plate in a porous medium. Int J Heat Mass Transf 44(15):2791-2799

8. Sharma PR, Singh G (2008) Unsteady MHD free convection and heat transfer along a vertical porous plate with variable suction and internal heat generation. Int J Appl Math Mech 4(5):1-8

9. Takhar HS, Ganesan P, Ekambavahar K, Soundalgekar VM (1997) Transient free convection past a semi-infinite vertical plate with variable surface temperature. Int J Numer Methods Heat Fluid Flow 7:280-296

10. Soundalgekar VM, Gupta SK, Birajdar NS (1979) Effects of mass transfer and free convection effects on MHD Stokes problem for a vertical plate. Nucl Eng Des 53:309-346

11. Sacheti NC, Chandran P, Singh AK (1994) An exact solution for unsteady magnetohydrodynamics free convection flow with constant heat flux. Int Commun Heat Mass Transf 21:131-142

12. Shanker B, Kishan N (1997) The effects of mass transfer on the MHD flow past an impulsively started infinite vertical plate with variable temperature or constant heat flux. J Energy Heat Mass Transf 19:273-278

13. Elbashbeshy EMA (1997) Heat and Mass transfer along a vertical plate with variable surface tension and concentration in the presence of the magnetic field. Int J Eng Sci 34(5):515-522

14. Helmy KA (1998) MHD unsteady free convection flow past a vertical porous plate. ZAMM 78:255-270

15. Takhar HS, Chamkha AJ, Nath G (2003) Unsteady mixed convection flow from a rotating vertical cone with a magnetic field. Heat Mass Transf 39:297-304

16. Ganesan P, Palani G (2004) Numerical solution of unsteady MHD flow past a semi-infinite isothermal vertical plate. In: Proceedings of the sixth ISHMT/ASME heat and mass transfer conference and seventeenth national heat and mass transfer conference, Kalpakkam, India, January 5-7, pp 184-187

17. Eastman JA, Choi SUS, Li S, Yu W, Thompson LJ (2001) Anomalously increased effective thermal conductivities of ethylene glycol-based nanofluids containing copper nanoparticles. Appl Phys Lett 78:718-720

18. Shirazi M, Shateri A, Bayareh M (2018) Numerical investigation of mixed convection heat transfer of a nanofluid in a circular enclosure with a rotating inner cylinder. J Therm Anal Calorim 133(2):1061-1073 
19. Bayareh M, Hajatzadeh Pordanjani A, Ahmadi Nadooshan A, Shiryan Dehkordi K (2016) Numerical study of the effects of stator boundary conditions and blade geometry on the efficiency of a scraped surface heat exchanger. Appl Therm Eng 113:1426-1436

20. Wang X, Yan X, Gao N, Chen G (2019) Prediction of thermal conductivity of various nanofluids with ethylene glaycol using artificial neural network. J Therm Sci. https://doi.org/10.1007/ s11630-019-1158-9

21. Arshad A, Jabbal M, Yan $Y$ (2019) A review on graphene based nanofluids: preparation, characterization and applications. J Mol Liq 279:444-484
22. Fu R, Liu Z, Chen Y (2019) Experimental investigation of turbulent forced heat transfer of $\mathrm{Fe}_{3} \mathrm{O}_{4}$ ethylene glycol-water nanofluid with highly disaggregated particles. Therm Sci Eng Prog 10:1-9

23. Jahanbakhshi A, Ahmadi Nadooshan A, Bayareh M (2018) Magnetic field effects on natural convection flow of a non-Newtonian fluid in an L-shaped enclosure. J Therm Anal Calorim 133:1407-1416

Publisher's Note Springer Nature remains neutral with regard to jurisdictional claims in published maps and institutional affiliations. 